\title{
Whispering-gallery-mode microbubble resonators: fabrication and characterization
}

\section{Amy Watkins}

Physics Department \& Tyndall National Institute, UCC

\section{Introduction}

Whispering is not an effective means of communication when a considerable distance separates the two conversationalists. In spite of this, a soft whisper can travel a very long way in the right environment - a whispering gallery. In 1910, the scientist Sir John William Strutt (Lord Rayleigh) witnessed this acoustic phenomenon in the Dome of St. Paul's Cathedral in London (see Fig. 1). Here, two people on opposite sides of the dome, up to 40 metres apart, can talk to each other by simply whispering against the curved wall. Inevitably, Lord Rayleigh - a true mathematician at heart - solved what he described as "The problem of the Whispering Gallery". He realised that, as the whisper travels along the curved surface, it loses very little energy and so can be heard after a great distance. Conversely, when the speaker talks at normal volume, the message possesses enough energy to complete several laps of the Dome. In this case, the sound waves overlap and the listener is unable to decipher the statement. If you have yet to visit such a gallery, I highly recommend it. A few other examples of this gallery include:

$\square$ The Echo Wall in the Temple of Heaven, Beijing;

$\square$ The Statuary Hall in the United States Capitol, Washington D.C.;

$\square$ The Gol Gumbaz in Bijapur, Karnataka State, India (see Fig. 1).

\section{Traditional microresonators}

Imagine shrinking the Whispering Gallery down to one-fifth of the size of the full-stop at the end of this sentence. Now, replace the sound waves with light and you have an optical microresonator. Traditionally, these microresonators take the form of solid glass disks, toroids (doughnut-shape) or spheres (see Fig. 2). For a good quality resonator, there are two specific criteria that need to be satisfied:

No defects, i.e., smooth surface;

Low ellipticity, i.e., as circular or spherical as possible. 


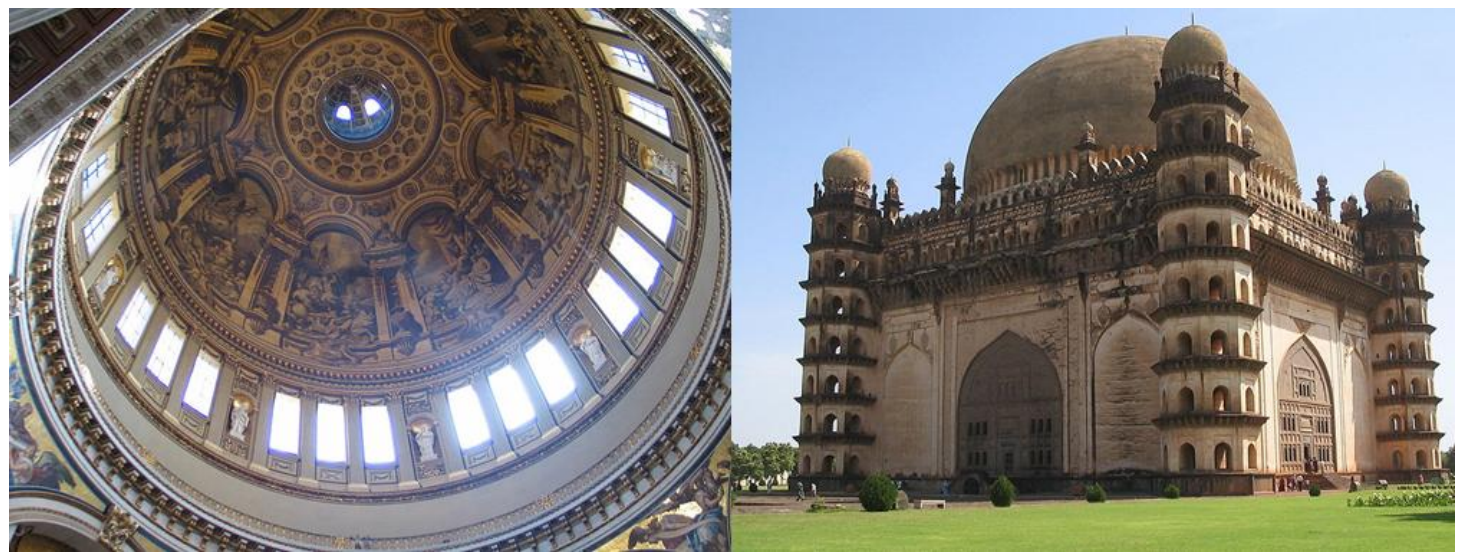

Figure 1: Examples of a Whispering Gallery. The Dome of 's Cathedral in (left) and the Gol Gumbaz in Bijapur (right).

The confinement of light in such a small volume leads to very high circulating intensities. The light completes several laps in precisely the same way as the normal volume sound waves in the Whispering Gallery. The exact number of laps depends on the quality of the resonator. In a good resonator, the light can easily complete tens of thousands of laps or reflections in real time.

My research involves the investigation of these novel optics devices to study the fundamental interactions of light and nanoparticles, e.g., atoms. Naturally, this work also extends to studying the sensing applications of these resonators.

\section{Coupling light into a microresonator}

The most effective process of inserting light into a microresonator is to place it in very close proximity to a tapered optical fibre, i.e., coupling. A tapered optical fibre is fabricated from standard optical fibre. In the modern world, standard fibre is widely used in a diverse range of applications from broadband connections used in telecommunications to

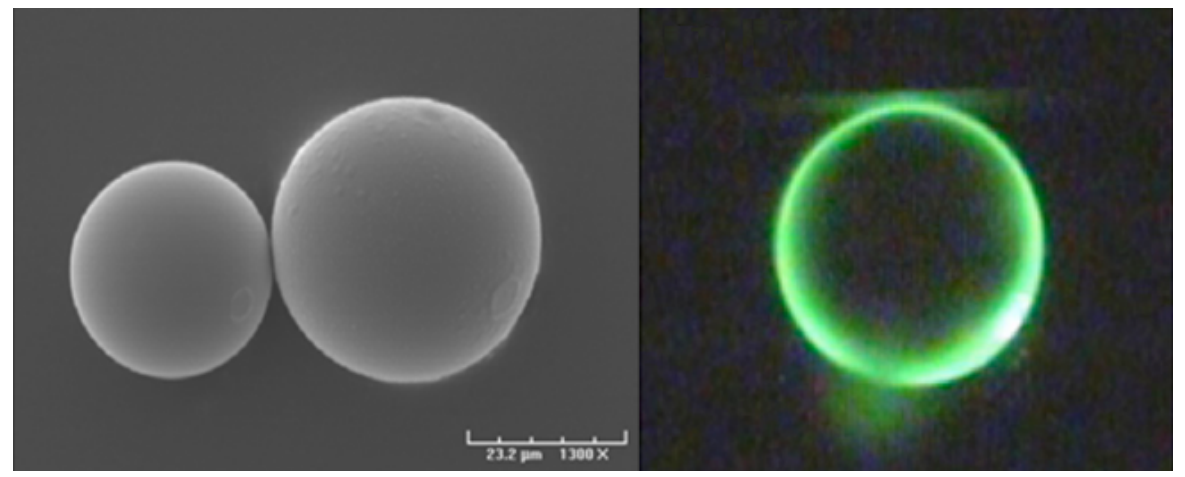

Figure 2: Example of a traditional resonator — solid glass microspheres (left) and light travelling inside a sphere (right). 

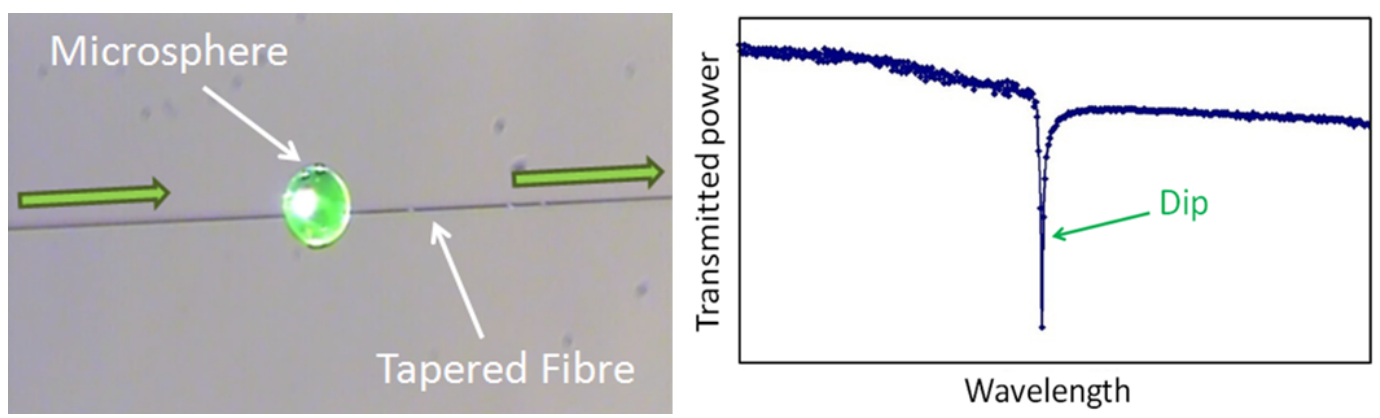

Figure 3: Microsphere and tapered optical fibre system (left) and whispering-gallery-mode (WGM) spectrum (right).

endoscopes in medical imaging and novelty Christmas decorations, to name but a few. In all of these examples, the light is confined to travelling inside the optical fibre.

For coupling into a microresonator, it is essential for the light to travel outside the fibre. This is achieved by placing the standard fibre into an oxygen-butane flame, where two translation stages (i.e., micro-positioning tables) pull it in opposite directions, thereby reducing the diameter of the fibre. This process is called tapering. After tapering, the midsection of the fibre is reduced in size until it becomes too small to contain the light. At this section, called the waist, the fibre now resembles spiders' silk in diameter. Here, the light travels briefly outside the fibre in the surrounding medium. This external light is called an evanescent field. It is via this field that effortless coupling of light into a resonator is facilitated. The waist is still connected to standard fibre at each end. After the waist, the light is directed to travel inside the standard fibre again, where it can be easily detected at the fibre end.

The sphere is placed in contact with the waist of a tapered fibre (see Fig. 3) and light from a laser is launched into the fibre. When the light reaches the waist, under very certain conditions, it couples into the sphere whereupon it completes several reflections, and then couples back out into the fibre. The condition where this coupling occurs is called "resonance" and is achieved by varying the wavelength (i.e., the colour) of the laser light. When the wavelength matches the size of the sphere, a dip is observed in the transmitted power of the light at the fibre end (see Fig. 3). These dips are also known as whispering-gallery-modes (WGM), which take their name from their acoustic analogue.

WGM resonators have been used comprehensively in a wide range of applications from the applied, i.e., biological of chemical sensing or laser engineering, to fundamental physics, in cavity-quantum electrodynamics experiments and cavity optomechanics. Primarily, these resonators can be exploited as extremely efficient lasers because a significantly larger amount of light is detected out of the resonator than is coupled in to it. This is a consequence of the unique characteristic that the light undergoes several reflections in such a small volume.

WGM resonators can also be used as extremely efficient sensors for a number of different 


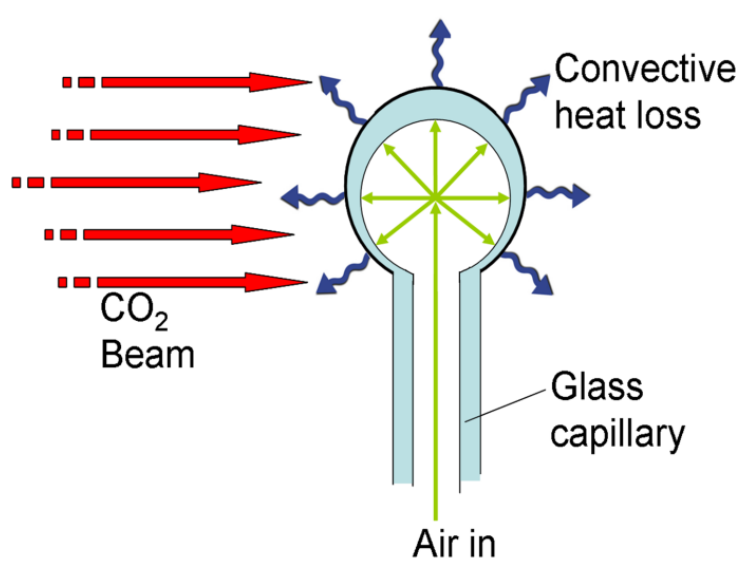

Figure 4: Diagram of optical microbubble resonator formation.

parameters, e.g., changes in pressure and temperature, a measure of mechanical vibrations, and the detection of biochemical samples, atoms or vapours. In biochemical sensing, even the presence of a single nanoparticle, such as an influenza particle, will disturb the light travelling inside the sphere. This disturbance is significantly magnified due to the number of reflections in the sphere. As a result, a change in the WGM spectrum is easily detected at the fibre end in the form of a shift in the position of the WGMs.

\section{My research}

As a sensing appliance, with the solid microsphere and tapered fibre system, the sample under investigation is required to reside outside the sphere. This can be challenging as the sample can substantially affect the coupling efficiency of the resonator with the tapered fibre. However, we can fabricate a new hollow glass microresonator, a microbubble, which has the distinct advantage that the sample can now be placed inside the cavity. This is a recent concept, of which there are only a few examples of analogous work, to our knowledge. The main aims of this work are to fabricate and characterize microbubbles in terms of their optical properties using methods comparable to that of the already welldeveloped solid microspheres.

\section{Microbubble Fabrication}

The microbubble fabrication process is similar to glass-blowing but on the microscopic scale with a laser as the heat source. A hollow glass capillary tube is tapered in the flame until it breaks. The tapered tip is gently heated using a $\mathrm{CO}_{2}$ laser to close it. The other end of the capillary is connected to an air pump which pressurizes the tube as the tip closes. When the tip is closed, the air pressure forces the soft glass tip to expand and blow out a bubble (see Fig. 4). 

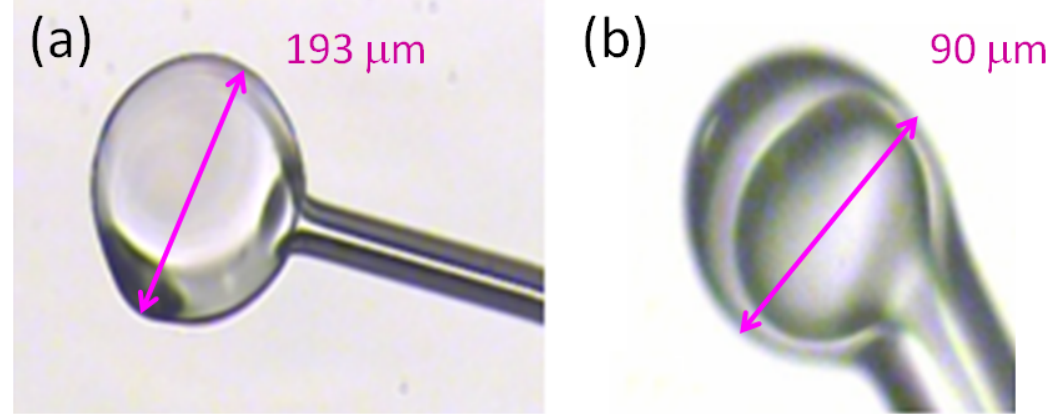

Figure 5: Images of microbubbles (a) when expansion is uncontrolled and (b) when expansion is controlled. The size of the bubble in (b) is similar to that of the microspheres.

If there is sufficient heating, the expansion of the bubble speeds up as the glass wall becomes thinner. The expansion then stops suddenly because the rate of heat losses at the bubble surface exceeds the heating rate from the laser. The resulting bubble (see Fig. 5a) will tend to have excess material on the surface which adds a distortion to the shape.

In order to make a more symmetrical bubble with a thicker wall, the heating of the bubble is stopped before the period of rapid expansion. During this process, the inside of the sphere filling with air is carefully monitored and the laser is manually controlled so that the expansion does not accelerate. This bubble (see Fig. 5b) is placed in contact with a tapered fibre using the same technique as for the sphere and corresponding WGMs are detected out the end of the fibre. The diameter of this bubble is similar in size to that of the microspheres.

\section{Microbubble Characterization}

To test if the light is penetrating into the hollow cavity of the bubble, it is filled with water. Initially, the WGM spectrum is recorded when the bubble is empty. The end of the capillary tube is connected to a syringe and a computer-controlled syringe pump. The air is pulled out of the bubble, to avoid a build-up of pressure, before water is pushed up the capillary tube into the cavity. The WGM spectrum is recorded again when the bubble is full of water. A shift in the position of the modes is observed (see Fig. 6). This is due to a difference in the refractive index between air and water, i.e., the light travels at a slightly different speed in each of the two media, which results in the position shift. When the bubble is emptied, the modes return to their original positions and this is a strong confirmation that these resonators are reusable. The resultant WGM shift is unique which can be used to identify the particular species under investigation, using a technique called refractive index sensing. 


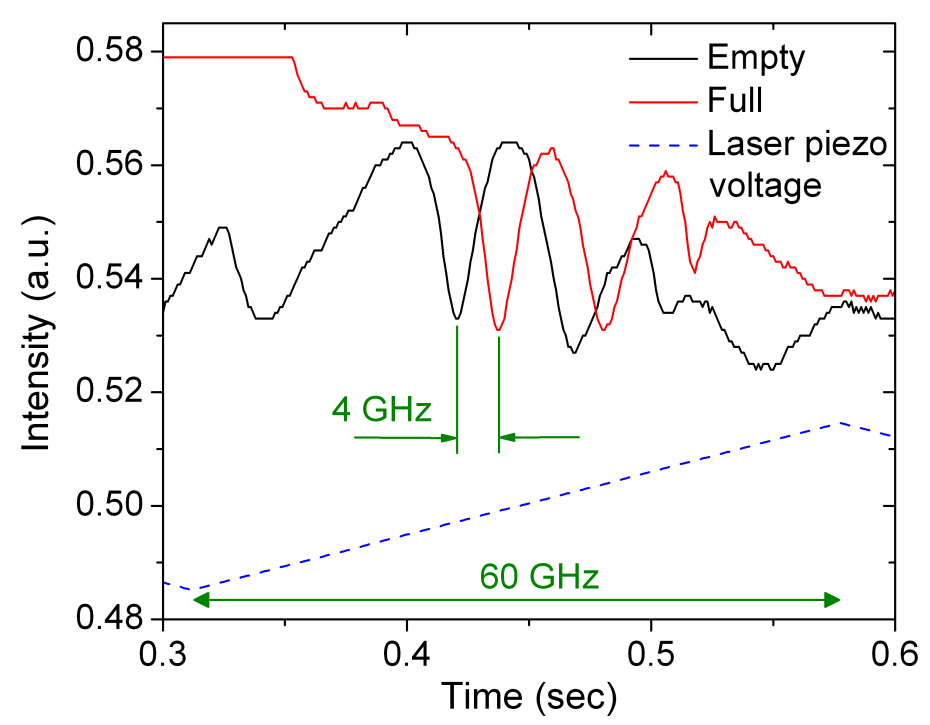

Figure 6: WGM spectra when bubble is empty (black trace) and full with water (red trace).

\section{Conclusion}

The fabrication and characterization of a new optical microresonator are described. These promising new resonators could find as many applications as their solid counterparts, especially in biochemical sensing. It is suggested that single nanoparticles smaller than an influenza particle can be detected using these novel devices. In context, well-developed optical microbubbles could provide the rapid detection of viral particles responsible for highly-infectious diseases, e.g., Anthrax, Ebola fever and Severe Acute Respiratory Syndrome (SARS), to suggest but a few. The early diagnosis of such potentially lethal infections is vital for successful treatment and prevention. Future work will involve further testing of the sensing capabilities of microbubbles.

Amy Watkins is a student in the Quantum Optics Group in the Physics Department and Tyndall National Institute under the supervision of Dr. Síle Nic Chormaic. The author would like to acknowledge the contribution of Dr. Jonathan Ward to this research and funding from the Irish Research Council for Science, Engineering \& Technology under the Embark Initiative 2008-2011. 